\title{
Evaluation of chromosomal abnormalities and copy number variations in fetuses with ultrasonic soft markers
}

\author{
Meiying Cai ${ }^{\dagger}$, Na Lin ${ }^{\dagger}$, Xuemei Chen, Meimei Fu, Nan Guo, Liangpu Xu ${ }^{*}$ and Hailong Huang ${ }^{*}$
}

\begin{abstract}
Background: Some ultrasonic soft markers can be found during ultrasound examination. However, the etiology of the fetuses with ultrasonic soft markers is still unknown. This study aimed to evaluate the genetic etiology and clinical value of chromosomal abnormalities and copy number variations (CNVs) in fetuses with ultrasonic soft markers.

Methods: Among 1131 fetuses, 729 had single ultrasonic soft marker, 322 had two ultrasonic soft markers, and 80 had three or more ultrasonic soft markers. All fetuses underwent conventional karyotyping, followed by single nucleotide polymorphism (SNP) array analysis.

Results: Among 1131 fetuses with ultrasonic soft markers, 46 had chromosomal abnormalities. In addition to the 46 fetuses with chromosomal abnormalities consistent with the results of the karyotyping analysis, the SNP array identified additional 6.1\% (69/1131) abnormal CNVs. The rate of abnormal CNVs in fetuses with ultrasonic soft marker, two ultrasonic soft markers, three or more ultrasonic soft markers were $6.2 \%, 6.2 \%$, and $5.0 \%$, respectively. No significant difference was found in the rate of abnormal CNVs among the groups.

Conclusions: Genetic abnormalities affect obstetrical outcomes. The SNP array can fully complement conventional karyotyping in fetuses with ultrasonic soft markers, improve detection rate of chromosomal abnormalities, and affect pregnancy outcomes.
\end{abstract}

Keywords: Single nucleotide polymorphism array analysis, Ultrasonic soft markers, Copy number variations, Fetus

\section{Background}

With the rapid development of high-resolution ultrasound and prenatal ultrasound diagnostic techniques, some ultrasonic soft markers can be found during ultrasound examination [1]. These ultrasonic soft markers include thickened nuchal translucency, ventriculomegaly, absent nasal bone, hyperechogenic bowel, choroid plexus cyst, short femur, echogenic intracardiac focus, mild tricuspid regurgitation, pyelectasis, single umbilical artery,

\footnotetext{
*Correspondence: xiliangpu@fmu.edu.cn; huanghailong@fmu.edu.cn ${ }^{\dagger}$ Meiying Cai and Na Lin contributed equally to this work Department of the Prenatal Diagnosis Center, Fujian Maternity and Child Health Hospital, Affiliated Hospital of Fujian Medical University, Fujian Key Laboratory for Prenatal Diagnosis and Birth Defect, Fuzhou, China
}

etc. In recent years, studies found an increased risk of chromosomal abnormalities in fetuses with ultrasonic soft markers. However, ultrasonic soft markers mainly refer to a non-specific index, which does not completely indicate the structural abnormality of the fetus, and may be a normal variation [2]. However, whether the fetus with ultrasonic soft markers should be diagnosed by prenatal diagnosis is still debated.

To explore the relationship among ultrasonic soft markers, genetic abnormalities in fetus, and its influence on pregnancy outcome, we systematically analyzed the relationship between ultrasonic soft markers and genetic abnormalities by observing 1131 fetuses with ultrasonic soft markers, aiming at expounding the utility of SNP array in fetus with ultrasonic soft markers. 


\section{Methods}

\section{Retrospective samples}

From November 2016 to July 2019, 1131 fetuses with ultrasonic soft markers underwent prenatal examination with consent after proper counseling at the Prenatal Diagnosis Center of the Fujian Provincial Maternal and Children Health Hospital. The mean maternal age was 28.9 (18-47) years, and the mean gestational week was 24.3 (13-38) weeks. Ultrasonic soft markers were diagnosed according to Li Shengli's diagnostic criteria [3]. The inclusion criteria for ultrasonic soft markers were as follows: thickened nuchal translucency, ventriculomegaly, absent nasal bone, hyperechogenic bowel, choroid plexus cyst, right subclavian vagus, short femur, echogenic intracardiac focus, mild tricuspid regurgitation, pyelectasis, single umbilical artery, and alteration of wave $A$ in the ductus venosus. The exclusion criteria were ultrasonic structural malformation and gemellary pregnancy. According to the number of ultrasonic soft markers, the fetuses were divided into a single ultrasonic soft marker group, two ultrasonic soft markers group, and three or more ultrasonic soft markers group.

Fetal samples were collected by chorionic villus sampling $(n=20)$, amniocentesis $(n=783)$, and cord blood sampling $(n=328)$ according to the gestational age. The villus was collected by chorionic villus sampling at 13-15 gestational weeks, amniotic fluid was collected by amniocentesis at 16-24 gestational weeks, and umbilical cord blood was collected by cordocentesis after the 24th gestational week.

The study was approved by the Fujian Provincial Maternal and Child Health Hospital regarding ethical conduct of research.

\section{Conventional karyotyping}

Fetal sample cells were cultured and analyzed by conventional karyotyping with Giemsa banding at a resolution of 450-550 bands.

\section{SNP array}

Digestion, amplification, purification, fragmentation, labeling, hybridization with chips, washing, scanning, and data analysis of sample genomic DNA were carried out according to the operation manual provided by Affymetrix Company of the United States. Chromosome Analysis Suite software 3.3 (Affymetrix, Santa Clara, CA, USA). and annotated based on genome version GRCh37 (hg19) were used to analyze the results. The referenced databases included the Database of Genomic Variants (DGV), Database of genomic variation and Phenotype in Humans using Ensembl Resources (DECIPHER), Online Mendelian Inheritance in Man (OMIM), International Standards for Cytogenomic Arrays (ISCA), and Global
Affymetrix User Pathology Shared Database (CGDB). According to the American College of Medical Genetics (ACMG) guidelines [4], CNVs were classified as being pathogenic, benign, or variations of uncertain clinical significance (VUS). All abnormal CNVs were verified by fluorescence in situ hybridization. Parental testing was carried out for fetuses who had abnormal CNVs to determine inheritance pattern. The microarray data from this study were submitted to the Gene Expression Omnibus repository (https://www.ncbi.nlm.nih.gov/geo/query/acc. cgi?acc $=$ GSE163799).

\section{Statistical analyses}

Statistical analyses were performed using SPSS Statistics 20 software (IBM Corp., Armonk, NY). P value of $<0.05$ indicated statistical significance. Chi-square test was used to analyze the detection rate of chromosomal abnormalities and abnormal CNVs among the ultrasonic soft markers groups.

\section{Results \\ Fetal profile}

There were 1131 fetuses with ultrasonic soft markers, including 729 fetuses with a single ultrasonic soft marker, 322 fetuses with two ultrasonic soft markers, and 80 fetuses with three or more ultrasonic soft markers. In 729 fetuses with a single ultrasonic soft marker, 302 had thickened nuchal translucency, 115 had ventriculomegaly, 76 had absent nasal bone, 65 fetuses had hyperechogenic bowel, 54 had choroid plexus cyst, 32 had right subclavian vagus, 26 had short femur, 22 had echogenic intracardiac focus, 16 had mild tricuspid regurgitation, 8 had pyelectasis, 7 had single umbilical artery, and 6 had alteration of wave $\mathrm{A}$ in the ductus venosus (Table 1).

\section{Conventional karyotyping}

Conventional karyotyping showed 46 fetuses with chromosome abnormalities, including 35 aneuploid, two mosaic chromosomal number abnormalities, and nine chromosomal structural abnormalities. Moreover, 35 fetuses had aneuploidy, including 23 fetuses with trisomy 21 , five fetuses with trisomy 18 , three fetuses with trisomy 13, two fetuses with 47, XXY, and two fetuses with 45 , X. In nine fetuses, the chromosomal structural abnormalities were as follows: 46,XX,del(5)(5p15.3), 46,XX,del(18) (p11.2), 46,XX, $\operatorname{add}(5)(\mathrm{p} 15.3), \quad 46, \mathrm{XY}, \operatorname{add}(4)(\mathrm{q} 35)$, $46, X X, \operatorname{add}(X)(q 21), \quad 46, X X, \operatorname{add}(10)(q 26), \quad 46, X Y, \operatorname{add}(2)$ (q37), 46,XX, add(12)(p13.3), and 46,XX,del(1)(q21).

Among the 46 fetuses with chromosomal abnormalities, 26 had single ultrasonic soft marker, 13 had two ultrasonic soft markers, and seven had three or more ultrasonic soft markers. The rates of chromosomal abnormalities in fetuses with single ultrasonic soft marker, two 
Table 1 Phenotypic characteristics of 1131 fetuses with ultrasonic soft markers

\begin{tabular}{ll}
\hline Classification of fetuses with ultrasonic soft markers & $\begin{array}{l}\text { Number } \\
\text { of fetuses }\end{array}$ \\
\hline Single ultrasonic soft marker & 729 \\
Thickened nuchal translucency & 302 \\
Ventriculomegaly & 115 \\
Absent nasal bone & 76 \\
Hyperechogenic bowel & 65 \\
Choroid plexus cyst & 54 \\
Right subclavian vagus & 32 \\
Short femur & 26 \\
Echogenic intracardiac focus & 22 \\
Mild tricuspid regurgitation & 16 \\
Pyelectasis & 8 \\
Single umbilical artery & 7 \\
Alteration of wave A in the ductus venosus & 6 \\
Two ultrasonic soft markers & 322 \\
Three or more ultrasonic soft markers & 80 \\
\hline
\end{tabular}

ultrasonic soft markers, and three or more ultrasonic soft markers were $3.6 \%(26 / 729), 4.0 \%(13 / 322)$, and $8.8 \%$ (7/80), respectively (Table 2$)$. No significant difference was found in the rate of chromosomal abnormalities among the ultrasonic soft marker groups $\left(\mathrm{X}^{2}=4.965\right.$, $P=0.084)$.

\section{SNP array results}

In addition to the 46 fetuses with chromosomal abnormalities consistent with the results of the conventional karyotyping, the SNP array identified additional $6.1 \%$
(69/1131) abnormal copy number variations (CNVs). Of the 69 abnormal CNVs, 37 were pathogenic CNVs and 32 were VUS. The rate of abnormal CNVs in fetuses with single ultrasonic soft marker, two ultrasonic soft markers, three or more ultrasonic soft markers were $6.2 \%$ (45/729), 6.2\% (20/322), and 5.0\% (4/80), respectively. No significant difference was found in the rate of abnormal CNVs among the fetuses with ultrasonic soft markers $\left(X^{2}=0.183, P=0.913\right)$ (Table 3). Abnormal CNVs were between 0.2 and 5.6 MB in size. Among the 37 pathogenic CNVs, nine were known microdeletion/microduplication syndrome, namely, four cases of 22q11.2 microdeletion syndrome, three cases of 22q11.2 microduplication syndrome, one case of 3q29 microdeletion syndrome, and one case of 7q11 microdeletion syndrome (Williams-Beuren syndrome). Thirty-two fetuses had VUS CNVs, including 21 fetuses with duplications, seven fetuses with deletions, and four fetuses lacked heterozygosity. VUS CNVs were associated with duplications of 16p13.11, 15q13.3, 2p16.1, 19q13.42, Xq23, 10q21.1, 2q36.1q36.2, 16p13.13p13.12, 8p23.2, 20q13.2, 2p22.3, 10q24.31q24.32, 13q14.3, 6q21; deletions of 15q11.2, 3p22.1, 9q31.3, 3p26.31, and 14q21.2q21.3 (Table 4).

The rates of chromosomal abnormalities and abnormal CNVs in fetuses with short femur $(23.1 \%, 6 / 26)$ were high, followed by those with mild tricuspid regurgitation $(12.5 \%, 2 / 16)$, thickened nuchal translucency $(12.0 \%, 36 / 302)$, choroid plexus cyst $(11.1 \%$, $6 / 54)$, absent nasal bone $(9.2 \%, 7 / 76)$, ventriculomegaly $(8.7 \%, 10 / 115)$, hyperechogenic bowel $(4.6 \%, 3 / 65)$, and echogenic intracardiac focus $(4.5 \%, 1 / 22)$. No chromosomal abnormalities and abnormal CNVs of the right

Table 2 Ultrasonic soft markers and conventional karyotyping

\begin{tabular}{|c|c|c|c|c|c|c|c|c|c|}
\hline Groups & $\begin{array}{l}\text { Number } \\
\text { of fetuses }\end{array}$ & $\begin{array}{l}\text { Rate } \\
\text { of chromosomal } \\
\text { abnormalities } \\
\text { (\%) }\end{array}$ & Trisomy 21 & Trisomy 18 & Trisomy 13 & $47, X X Y$ & $45, X$ & $\begin{array}{l}\text { Mosaic } \\
\text { chromosomal } \\
\text { number } \\
\text { abnormalities }\end{array}$ & $\begin{array}{l}\text { Chromosomal } \\
\text { structural } \\
\text { abnormalities }\end{array}$ \\
\hline $\begin{array}{l}\text { Single ultrasonic } \\
\text { soft marker }\end{array}$ & 729 & $26(3.6)$ & 12 & 3 & 3 & 1 & 2 & & 5 \\
\hline Short femur & 26 & $2(7.7 \%)$ & & & & & & & 2 \\
\hline $\begin{array}{l}\text { Thickened nuchal } \\
\text { translucency }\end{array}$ & 302 & $18(6.0 \%)$ & 10 & 1 & 3 & & 1 & & 3 \\
\hline $\begin{array}{l}\text { Choroid plexus } \\
\text { cyst }\end{array}$ & 54 & $2(3.7 \%)$ & & 2 & & & & & \\
\hline Absent nasal bone & 76 & $2(2.6 \%)$ & 1 & & & 1 & & & \\
\hline Ventriculomegaly & 115 & $2(1.7 \%)$ & 1 & & & & 1 & & \\
\hline $\begin{array}{l}\text { Two ultrasonic soft } \\
\text { markers }\end{array}$ & 322 & $13(4.0 \%)$ & 7 & 1 & & 1 & & 1 & 3 \\
\hline $\begin{array}{l}\text { Three or more } \\
\text { ultrasonic soft } \\
\text { markers }\end{array}$ & 80 & $7(8.8 \%)$ & 4 & 1 & & & & 1 & 1 \\
\hline
\end{tabular}


Table 3 Ultrasonic soft markers and SNP array

\begin{tabular}{lcccc}
\hline Groups & Number of fetuses & $\begin{array}{l}\text { Number of abnormal } \\
\text { CNVs }\end{array}$ & Pathogenic CNVs & VUS CNVs \\
\hline Single ultrasonic soft marker & 729 & $45(6.2 \%)$ & 24 & 21 \\
Short femur & 26 & $4(15.4 \%)$ & 3 & 1 \\
Mild tricuspid regurgitation & 16 & $2(12.5 \%)$ & 1 & 1 \\
Choroid plexus cyst & 54 & $4(7.4 \%)$ & 4 & 3 \\
Ventriculomegaly & 115 & $5(7.0 \%)$ & 2 & 4 \\
Absent nasal bone & 76 & $18(6.6 \%)$ & 2 & 3 \\
Thickened nuchal translucency & 302 & $3(4.6 \%)$ & 1 & 1 \\
Hyperechogenic bowel & 65 & $1(4.5 \%)$ & 10 & 1 \\
Echogenic intracardiac focus & 22 & $20(6.2 \%)$ & 3 & 1 \\
Two ultrasonic soft markers & 322 & $4(5.0 \%)$ & 1 \\
Three or more ultrasonic soft markers & 80 & & 1
\end{tabular}

CNVs abnormal copy number variations, SNP single nucleotide polymorphism, VUS variation of uncertain clinical significance

subclavian vagus, pyelectasis, single umbilical artery, and alteration of wave $\mathrm{A}$ in the ductus venosus were detected.

\section{Inheritance analysis and pregnancy outcome}

We screened inheritance information from nine cases of abnormal karyotype results (exempt 35 cases of aneuploid and two cases of mosaic chromosomal number abnormalities) and 50 cases of abnormal CNVs (exempt the parents refused genetic testing in 19 cases). Inheritance analysis showed that nine fetuses with abnormal CNVs were inherited from unaffected parents, and 50 (nine fetuses with abnormal karyotype results and 40 fetuses with abnormal CNVs) were de novo CNVs. Among 1131 fetuses with ultrasonic soft markers, 1108 fetuses were successfully followed, except for 23 fetuses who were loss to follow-up. Pregnancies of 46 fetuses with chromosomal abnormalities, 37 fetuses with pathogenic CNVs, and three fetuses with VUS CNVs were terminated. Parents of the 29 fetuses with VUS CNVs chose to continue pregnancy, and newborns were followed after birth. Among 29 fetuses with VUS CNVs, one died of unknown cause after birth, two had delayed development after birth, one was lost follow-up, and 25 fetuses were normal development and growth.

\section{Discussion}

In this study, 1131 cases with ultrasonic soft markers were observed as indication for prenatal diagnosis, including 729 fetuses with a single ultrasonic soft marker, 322 fetuses with two ultrasonic soft markers, and 80 fetuses with three or more ultrasonic soft markers. These 1131 fetuses with ultrasonic soft markers were analyzed by conventional karyotyping, and the detection rate of conventional karyotyping was $4.1 \%$.
In addition to the 46 fetuses with chromosomal abnormalities consistent with the results of the conventional karyotyping, the SNP array identified additional 6.1\% abnormal CNVs.

Trisomy 21 and chromosomal structural abnormalities were the most common chromosomal abnormalities in fetuses with ultrasonic soft markers, accounting for $50 \%(23 / 46)$ and $20 \%(9 / 46)$, followed by trisomy 18 , trisomy 13, and other chromosomal number abnormalities. The rate of chromosomal abnormalities in fetuses with single ultrasonic soft marker, two ultrasonic soft markers, and three or more ultrasonic soft markers were $3.6 \%, 4.0 \%$, and $8.8 \%$, respectively. No significant difference was found in the detection rate of chromosomal abnormalities among fetuses with ultrasonic soft markers. This is obviously different from previous reports that clusters of ultrasonic soft markers greatly increased the likelihood of chromosomal abnormalities compared with a single ultrasonic soft marker $[5,6]$. This difference may be related to the selected cohort of fetuses with ultrasonic soft markers.

Among 1131 fetuses with ultrasonic soft markers, in addition to the 46 fetuses with chromosomal abnormalities consistent with the results of karyotyping analysis, the SNP array also identified abnormal CNVs, and no significant difference was found in the detection rate of abnormal CNVs among fetuses with ultrasonic soft markers. Previous studies [7-10] have reported that SNP array was used in fetuses with ultrasonic structural abnormalities, and there are few reports in which SNP array is used in fetuses with ultrasonic soft markers. We used SNP array to detect abnormal CNVs in fetuses with ultrasonic soft markers, and the SNP array results showed that the detection rate of abnormal CNVs was slightly higher than the previous literature [11]. 
Table 4 Abnormal CNVs in fetuses with ultrasonic soft marker

\begin{tabular}{|c|c|c|c|c|c|c|}
\hline Case & Indication & SNP array & Interpretation & Size $\mathrm{Mb}$ & Outcome & Inheritance \\
\hline 1 & Short femur & $\begin{array}{l}\operatorname{arr}[\mathrm{hg} 19] \\
\quad 17 \mathrm{q} 21.31(41,774,473-42,491,805) \times 4\end{array}$ & $P$ & 0.7 & TP & Denovo \\
\hline 2 & Short femur & $\begin{array}{l}\operatorname{arr}[\mathrm{hg} 19] 10 \mathrm{q} 21.3(68,972,662- \\
\quad 69,925,900) \times 1\end{array}$ & $P$ & 0.9 & TP & Denovo \\
\hline 3 & Short femur & $\begin{array}{l}\operatorname{arr}[\mathrm{hg} 19] 4 \mathrm{q} 28 \\
\quad .3 \mathrm{q} 31.3(133,718,289-154,569,367) \mathrm{hmz}\end{array}$ & VUS & 20.8 & $\mathrm{TD}$ & - \\
\hline 4 & Short femur & $\begin{array}{l}\operatorname{arr}[\text { hg19] 15q13.3(32,003,537- } \\
\quad 32,444,043) \times 3\end{array}$ & VUS & 0.4 & $\mathrm{TP}$ & Denovo \\
\hline 5 & Thickened nuchal translucency & $\begin{array}{l}\operatorname{arr}[\mathrm{hg} 19] \\
22 \mathrm{q} 11.21(18,648,855-21,459,713) \times 3\end{array}$ & $P$ & 2.8 & TP & Maternal \\
\hline 6 & Thickened nuchal translucency & $\begin{array}{l}\operatorname{arr}[\text { hg 19]Yq11.2 } \\
22 \text { q11.223(20,252,055-25,863,576) } \times 0\end{array}$ & $P$ & 5.6 & TP & Denovo \\
\hline 7 & Thickened nuchal translucency & $\begin{array}{l}\operatorname{arr}[\mathrm{hg} 19] 22 \mathrm{2q11} .21(18,648,855- \\
21,800,471) \times 3\end{array}$ & $p$ & 3.1 & TP & Denovo \\
\hline 8 & Thickened nuchal translucency & $\begin{array}{l}\text { arr[hg19] 3q29(195,678,474- } \\
\quad 197,340,833) \times 1\end{array}$ & $\mathrm{p}$ & 1.6 & TP & Denovo \\
\hline 9 & Thickened nuchal translucency & $\begin{array}{l}\operatorname{arr}[\text { hg19] 1p31.3(61,886,890- } \\
\quad 63,701,576) \times 1\end{array}$ & $P$ & 1.8 & $\mathrm{TP}$ & Denovo \\
\hline 10 & Thickened nuchal translucency & $\begin{array}{l}\operatorname{arr}[\mathrm{hg} 19] 22 \mathrm{2q} 11.21(20,730,143- \\
21,800,471) \times 3\end{array}$ & $P$ & 1.0 & $\mathrm{TP}$ & Denovo \\
\hline 11 & Thickened nuchal translucency & $\begin{array}{l}\operatorname{arr}[\mathrm{hg} 19] 1 \mathrm{q} 21 \\
\quad .1 \mathrm{q} 21.2(145,829,473-148,520,164) \times 1\end{array}$ & $P$ & 2.7 & $\mathrm{TP}$ & Denovo \\
\hline 12 & Thickened nuchal translucency & $\begin{array}{l}\operatorname{arr}[\mathrm{hg} 19] \\
\quad 22 \mathrm{q} 11.21(20,716,876-21,800,471) \times 1\end{array}$ & $p$ & 1.0 & $\mathrm{TP}$ & Denovo \\
\hline 13 & Thickened nuchal translucency & $\begin{array}{l}\text { arr[hg19] 16p13.11(14,910,158- } \\
\quad 16,508,123) \times 1\end{array}$ & $P$ & 1.6 & TP & Denovo \\
\hline 14 & Thickened nuchal translucency & $\begin{array}{l}\operatorname{arr}[\mathrm{hg} 19] \mathrm{Xp22.31(6,449,836-} \\
\quad 8,141,076) \times 0,(X Y) \times 1\end{array}$ & $P$ & 1.7 & TP & Denovo \\
\hline 15 & Thickened nuchal translucency & $\begin{array}{l}\operatorname{arr}[\text { hg 19] Xq28(153,560,562- } \\
\quad 153,826,362) \times 3\end{array}$ & $P$ & 0.3 & TP & Denovo \\
\hline 16 & Thickened nuchal translucency & $\begin{array}{l}\operatorname{arr}[\mathrm{hg} 19] 2 \mathrm{p} 16.1(55,993,333- \\
\quad 60,450,583) \times 3\end{array}$ & VUS & 4.5 & TD & - \\
\hline 17 & Thickened nuchal translucency & $\begin{array}{l}\operatorname{arr}[\mathrm{hg} 19] 19 \mathrm{q} 13.42(53,717,606- \\
\quad 54,656,213) \times 3\end{array}$ & VUS & 0.9 & TD & Denovo \\
\hline 18 & Thickened nuchal translucency & $\begin{array}{l}\operatorname{arr}[\text { hg19]Xq23(109,823,197- } \\
\quad 110,252,333) \times 3\end{array}$ & VUS & 0.4 & $\mathrm{TD}$ & - \\
\hline 19 & Thickened nuchal translucency & $\begin{array}{l}\operatorname{arr}[\mathrm{hg} 19] 15 \mathrm{q} 13.3(32,003,537- \\
\quad 32,444,043) \times 3\end{array}$ & VUS & 0.4 & $\mathrm{TD}$ & Denovo \\
\hline 20 & Thickened nuchal translucency & $\begin{array}{l}\operatorname{arr}[\mathrm{hg} 19] 10 \mathrm{q} 21.1(53,477,821- \\
\quad 55,138,122) \times 3\end{array}$ & VUS & 1.7 & $\mathrm{TD}$ & Denovo \\
\hline 21 & Thickened nuchal translucency & $\begin{array}{l}\operatorname{arr}[\text { hg19] 9q31.3(113,177,821- } \\
\quad 113,544,998) \times 1\end{array}$ & VUS & 0.4 & TD & - \\
\hline 22 & Thickened nuchal translucency & $\begin{array}{l}\operatorname{arr}[\mathrm{hg} 19] 1 \mathrm{q} 21 \\
.1 \mathrm{q} 21.2(144,077,593-148,750,533) \mathrm{hmz} \\
\operatorname{arr}[\mathrm{hg} 19] 3 \mathrm{p} 21 . \\
31 \mathrm{p} 21.1(48,166,782-53,172,233) \mathrm{hmz} \\
\operatorname{arr}[\mathrm{hg} 19] 5 \mathrm{q} 21 \\
.3 \mathrm{q} 22.1(107,196,975-110,478,806) \mathrm{hmz} \\
\operatorname{arr}[\mathrm{hg} 19] 12 \mathrm{q} 21 . \\
31 \mathrm{q} 21.33(82,446,525-91,707,400) \mathrm{hmz} \\
\operatorname{arr}[\mathrm{hg} 19] 14 \mathrm{q} 31 \\
.2 \mathrm{q} 32.12(84,339,970-92,755,472) \mathrm{hmz} \\
\operatorname{arr}[\mathrm{hg} 19] 15 \mathrm{q} 24 \\
.1 \mathrm{q} 25.3(73,065,223-87,467,262) \mathrm{hmz} \\
\operatorname{arr}[\mathrm{hg} 19] 16 \mathrm{p} 13.3(94,807-3,112,982) \mathrm{hmz} \\
\operatorname{arr}[\mathrm{hg} 19] 17 \mathrm{p} 12 \mathrm{p} 11.2(15,838,698- \\
22,170,994) \mathrm{hmz}\end{array}$ & VUS & 99 & $\mathrm{TD}$ & - \\
\hline 23 & Ventriculomegaly & $\begin{array}{l}\operatorname{arr}[\text { hg19] } \\
\quad 16 p 11.2(29,567,296-30,190,029) \times 1\end{array}$ & $P$ & 0.6 & TP & Denovo \\
\hline
\end{tabular}


Table 4 (continued)

\begin{tabular}{|c|c|c|c|c|c|c|}
\hline Case & Indication & SNP array & Interpretation & Size Mb & Outcome & Inheritance \\
\hline 24 & Ventriculomegaly & $\begin{array}{l}\operatorname{arr}[\mathrm{hg} 19] 5 \mathrm{q} 35 \\
.2 \mathrm{q} 35.3(175,416,095-177,482,506) \times 1\end{array}$ & $P$ & 2.0 & TP & Denovo \\
\hline 25 & Ventriculomegaly & 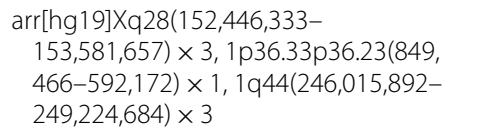 & $P$ & $1.1,7.7,3.2$ & TP & Denovo \\
\hline 26 & Ventriculomegaly & $\begin{array}{l}\operatorname{arr}[\mathrm{hg} 19 \\
\quad \text { ]1q21.1(145,375,770-145,770,627) } \times 1 \\
\quad 9 p 24.1(4,623,660-5,501,699) \times 3\end{array}$ & $P$ & $0.7,0.9$ & $\mathrm{TP}$ & Denovo \\
\hline 27 & Ventriculomegaly & $\begin{array}{l}\operatorname{arr}[\mathrm{hg} 19] 3 \mathrm{p} 22.1(42,875,130- \\
\quad 43,309,436) \times 1\end{array}$ & VUS & 0.4 & $\mathrm{TP}$ & Denovo \\
\hline 28 & Ventriculomegaly & $\begin{array}{l}\operatorname{arr}[\text { hg1 19]2q36 } \\
\quad .1 \mathrm{q} 36.2(224,459,152-225,330,583) \times 3\end{array}$ & VUS & 0.9 & $\mathrm{TD}$ & Denovo \\
\hline 29 & Ventriculomegaly & $\operatorname{arr}[$ hg19]3p26.3(1,855,754-2,663,625) × 1 & VUS & 0.8 & $\mathrm{TD}$ & - \\
\hline 30 & Ventriculomegaly & $\begin{array}{l}\operatorname{arr}[\mathrm{hg} 19] 16 \mathrm{p} 13.11(15,058,820- \\
\quad 16,309,046) \times 3\end{array}$ & VUS & 1.3 & $\mathrm{TD}$ & - \\
\hline 31 & Absent nasal bone & $\begin{array}{l}\operatorname{arr}[\mathrm{hg} 19] 15 \mathrm{q} 13 \\
\quad .2 \mathrm{q} 13.3(30,386,398-32,444,261) \times 1\end{array}$ & $P$ & 2.0 & $\mathrm{TP}$ & Denovo \\
\hline 32 & Absent nasal bone & $\begin{array}{l}\operatorname{arr}[\mathrm{hg} 19] \\
\quad 16 \mathrm{p} 12.2(21,816,542-22,710,614) \times 1\end{array}$ & $P$ & 1.0 & $\mathrm{TP}$ & Denovo \\
\hline 33 & Absent nasal bone & $\begin{array}{l}\operatorname{arr}[\mathrm{hg} 19] 15 q 13.3(32,021,609- \\
\quad 32,444,043) \times 3\end{array}$ & VUS & 0.4 & $\mathrm{TD}$ & Denovo \\
\hline 34 & Absent nasal bone & $\begin{array}{l}\operatorname{arr}[\text { hg19] 15q11.2(22,770,421- } \\
23,276,833) \times 1\end{array}$ & VUS & 0.5 & $\mathrm{TD}$ & Paternal \\
\hline 35 & Absent nasal bone & $\begin{array}{l}\operatorname{arr}[\mathrm{hg} 19] 16 \mathrm{p} 13 \\
\quad 13 \mathrm{p} 13.12(11,528,493-12,934,811) \times 3\end{array}$ & VUS & 1.4 & $\mathrm{TD}$ & Maternal \\
\hline 36 & Hyperechogenic bowel & $\begin{array}{l}\operatorname{arr}[\mathrm{hg} 19] \\
22 \mathrm{q} 13.33(49,683,904-51,197,766) \times 1\end{array}$ & $P$ & 3.1 & $\mathrm{TP}$ & Denovo \\
\hline 37 & Hyperechogenic bowel & $\begin{array}{l}\operatorname{arr}[\text { hg19]10q11. } \\
\quad 21 \mathrm{q} 11.22(42,433,738-48,006,310) \times 1\end{array}$ & $P$ & 5.5 & $\mathrm{TP}$ & Denovo \\
\hline 38 & Hyperechogenic bowel & $\begin{array}{l}\operatorname{arr}[\text { hg19] } \\
\quad 16 \mathrm{p} 13.11(15,171,146-16,309,046) \times 3\end{array}$ & VUS & 1.1 & TD & Paternal \\
\hline 39 & Choroid plexus cyst & $\begin{array}{l}\operatorname{arr}[\mathrm{hg} 19] \\
\quad 22 \mathrm{q} 11.21(18,648,855-21,800,471) \times 1\end{array}$ & $P$ & 3.1 & TP & Maternal \\
\hline 40 & Choroid plexus cyst & $\begin{array}{l}\operatorname{arr}[\mathrm{hg} 19] 15 \mathrm{q} 13.3(32,011,458- \\
\quad 32,444,043) \times 3\end{array}$ & VUS & 0.4 & TD & - \\
\hline 41 & Choroid plexus cyst & $\operatorname{arr}[$ hg19]8p23.2(3,703,883-5,940,433) × 3 & VUS & 2.2 & TD & - \\
\hline 42 & Choroid plexus cyst & $\begin{array}{l}\operatorname{arr}[\mathrm{hg} 19] 20 \mathrm{q} 13.2(53,545,723- \\
54,866,110) \times 3\end{array}$ & VUS & 1.3 & $\mathrm{TD}$ & Denovo \\
\hline 43 & Mild tricuspid regurgitation & $\begin{array}{l}\operatorname{arr}[\mathrm{hg} 19] 1 \mathrm{q} 21.1(145,287,532- \\
\quad 145,966,117) \times 3\end{array}$ & $P$ & 0.7 & $\mathrm{TP}$ & Paternal \\
\hline 44 & Mild tricuspid regurgitation & $\begin{array}{l}\operatorname{arr}[\mathrm{hg} 19] \\
\quad 16 \mathrm{p} 13.11(15,510,512-16,309,046) \times 3\end{array}$ & VUS & 0.8 & TD & - \\
\hline 45 & Echogenic intracardiac focus & $\begin{array}{l}\operatorname{arr}[\mathrm{hg} 19] 17 \mathrm{q} 12(34,440,088- \\
36,351,919) \times 3\end{array}$ & $P$ & 1.9 & $\mathrm{TP}$ & Paternal \\
\hline 46 & $\begin{array}{l}\text { Right subclavian vagus, echogenic intra- } \\
\text { cardiac focus }\end{array}$ & $\begin{array}{l}\operatorname{arr}[\mathrm{hg} 19] \\
\quad 22 \mathrm{q} 11.21(18,648,855-21,800,471) \times 1\end{array}$ & $P$ & 3.1 & TP & Denovo \\
\hline 47 & $\begin{array}{l}\text { Cavity of septum pellucidum, amniotic } \\
\text { fluid is excessive }\end{array}$ & $\begin{array}{l}\operatorname{arr}[\mathrm{hg} 19] 17 \mathrm{p} 12(14,099,504- \\
\quad 15,491,533) \times 1\end{array}$ & $P$ & 1.3 & $\mathrm{TP}$ & - \\
\hline 48 & Echogenic intracardiac focus, pyelectasis & $\begin{array}{l}\operatorname{arr}[\mathrm{hg} 19] 6 \mathrm{p} 21 \\
\quad 32 \mathrm{p} 21.31(32,965,747-35,234,269) \times 3\end{array}$ & $P$ & 2.2 & TP & Denovo \\
\hline 49 & $\begin{array}{l}\text { Absent nasal bone, echogenic intracardiac } \\
\text { focus }\end{array}$ & $\begin{array}{l}\operatorname{arr}[\mathrm{hg} 19] 17 \mathrm{p} 12(14,070,219- \\
15,484,335) \times 1\end{array}$ & $P$ & 1.4 & TP & Denovo \\
\hline 50 & Absent nasal bone, pyelectasis & $\begin{array}{l}\operatorname{arr}[\mathrm{hg} 19] 2 \mathrm{p} 22.3(34,002,379- \\
\quad 35,076,738) \times 3\end{array}$ & VUS & 1.0 & TD & - \\
\hline 51 & $\begin{array}{l}\text { Mild tricuspid regurgitation, pericardial } \\
\text { effusion }\end{array}$ & $\begin{array}{l}\operatorname{arr}[\mathrm{hg} 19] 5 \mathrm{p} 15 \\
\quad 33 \mathrm{p} 15.31(4,482,234-6,636,035) \times 1\end{array}$ & $P$ & 2.1 & $\mathrm{TP}$ & Denovo \\
\hline
\end{tabular}


Table 4 (continued)

\begin{tabular}{|c|c|c|c|c|c|c|}
\hline Case & Indication & SNP array & Interpretation & Size $\mathrm{Mb}$ & Outcome & Inheritance \\
\hline 52 & Short femur, hyperechogenic bowel & $\begin{array}{l}\operatorname{arr}[\mathrm{hg} 19] 10 \mathrm{q} 11 \\
\quad 22 \mathrm{q} 11.23(46,252,072-51,903,756) \times 1\end{array}$ & $P$ & 5.6 & TP & - \\
\hline 53 & $\begin{array}{l}\text { Mild tricuspid regurgitation, hyperecho- } \\
\text { genic bowel }\end{array}$ & $\begin{array}{l}\operatorname{arr}[\mathrm{hg} 19] \\
\quad 15 \mathrm{q} 11.2(22,770,421-23,286,423) \times 1\end{array}$ & VUS & 0.5 & TD & Paternal \\
\hline 54 & $\begin{array}{l}\text { Mild tricuspid regurgitation, echogenic } \\
\text { intracardiac focus }\end{array}$ & $\begin{array}{l}\operatorname{arr}[\text { hg19] 15q11.2(22,770,421- } \\
23,277,436) \times 1\end{array}$ & VUS & 0.5 & $\mathrm{TD}$ & Denovo \\
\hline 55 & $\begin{array}{l}\text { Mild tricuspid regurgitation, echogenic } \\
\text { intracardiac focus }\end{array}$ & $\begin{array}{l}\text { arr[hg19] Xp22.33 or Yp1 1.32(387,396- } \\
629,998 \text { or 337,396-579,998) × } 1\end{array}$ & $P$ & 0.2 & TP & Denovo \\
\hline 56 & $\begin{array}{l}\text { Echogenic intracardiac focus, ventriculo- } \\
\text { megaly }\end{array}$ & 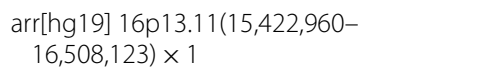 & $P$ & 1.0 & $\mathrm{TP}$ & Denovo \\
\hline 57 & $\begin{array}{l}\text { Single umbilical artery, mild tricuspid } \\
\text { regurgitation }\end{array}$ & $\begin{array}{l}\operatorname{arr}[\mathrm{hg} 19] 1 \mathrm{q} 21 \\
\quad .1 \mathrm{q} 21.2(145,084,525-147,885,600) \times 1\end{array}$ & $P$ & 2.8 & TP & Paternal \\
\hline 58 & $\begin{array}{l}\text { Echogenic intracardiac focus, mild tricus- } \\
\text { pid regurgitation }\end{array}$ & $\begin{array}{l}\operatorname{arr}[\mathrm{hg} 19] \text { Xp22.33 or Yp11.32(168,551- } \\
629,999 \text { or } 118,551-579,999) \times 1\end{array}$ & $P$ & 0.5 & TP & Denovo \\
\hline 59 & $\begin{array}{l}\text { Hyperechogenic bowel, mild tricuspid } \\
\text { regurgitation }\end{array}$ & $\begin{array}{l}\operatorname{arr}[\text { hg19] 16p13.11(15,058,820- } \\
\text { 16,309,046) ×3 }\end{array}$ & VUS & 1.25 & TD & Denovo \\
\hline 60 & $\begin{array}{l}\text { Hyperechogenic bowel, enlargement of } \\
\text { intestinal canal }\end{array}$ & $\begin{array}{l}\operatorname{arr}[\mathrm{hg} 19] 16 \mathrm{p} 13.11(14,900,042- \\
16,508,123) \times 3\end{array}$ & VUS & 1.6 & $\mathrm{TD}$ & Denovo \\
\hline 61 & Ventriculomegaly, choroid plexus cyst & $\begin{array}{l}\operatorname{arr}[\text { hg19]14q21 } \\
\quad .2 q 21.3(46,782,405-49,288,860) \times 1\end{array}$ & VUS & 2.5 & TP & - \\
\hline 62 & Ventriculomegaly, short femur & $\begin{array}{l}\operatorname{arr}[\mathrm{hg} 19] 3 \mathrm{q} 26 \\
.1 \mathrm{q} 29(163,256,369-197,791,601) \mathrm{hmz} \\
\operatorname{arr}[\mathrm{hg} 19] 5 \mathrm{p} 13 \\
.1 \mathrm{p} 11(41,029,137-46,313,469) \mathrm{hmz} \\
\operatorname{arr}[\mathrm{hg} 19] 6 \mathrm{q} 24 \\
.2 \mathrm{q} 25(143,341,406-161,527,784) \mathrm{hmz} \\
\operatorname{arr}[\mathrm{hg} 19] 12 \mathrm{q} 13 \\
.2 \mathrm{q} 21.2(56,011,100-77,134,151) \mathrm{hmz} \\
\operatorname{arr}[\mathrm{hg} 19] 17 \mathrm{q} 21 \\
.2 \mathrm{q} 21.32(39,639,602-45,479,706) \mathrm{hmz} \\
\operatorname{arr}[\mathrm{hg} 19] 21 \mathrm{q} 21 \\
.3 \mathrm{q} 22.2(28,124,165-42,352,287) \mathrm{hmz}\end{array}$ & VUS & 99.1 & TD & - \\
\hline 63 & $\begin{array}{l}\text { Ventriculomegaly, echogenic intracardiac } \\
\text { focus }\end{array}$ & $\begin{array}{l}\text { arr[hg19]10q24. } \\
31 \mathrm{q} 24.32(102,972,457-103,179,063) \times 3\end{array}$ & VUS & 0.2 & $\mathrm{TD}$ & Denovo \\
\hline 64 & Mild tricuspid regurgitation, pyelectasis & $\begin{array}{l}\operatorname{arr}[\text { hg19] } \\
\quad 13 q 14.3(52,649,105-53,172,866) \times 3\end{array}$ & VUS & 0.5 & $\mathrm{TD}$ & - \\
\hline 65 & $\begin{array}{l}\text { Thickened nuchal translucency, thickening } \\
\text { of skin in chest and abdomen }\end{array}$ & $\begin{array}{l}\operatorname{arr}[\mathrm{hg} 19] 1 \mathrm{p} \\
33 \mathrm{p} 32.3(50,051,514-53,274,566) \mathrm{hmz} \\
\operatorname{arr}[\mathrm{hg} 19] 2 \mathrm{q} 23 \\
.3 \mathrm{q} 24.1(153,771,280-158,783,675) \mathrm{hmz} \\
\operatorname{arr}[\mathrm{hg} 19] 3 \mathrm{q} 21 \\
.2 \mathrm{q} 22.1(124,817,983-129,317,745) \mathrm{hmz} \\
\operatorname{arr}[\mathrm{hg} 19] 3 \mathrm{q} 22 \\
.1 \mathrm{q} 23(133,262,566-139,418,898) \mathrm{hmz} \\
\operatorname{arr}[\mathrm{hg} 19] 3 \mathrm{q} 26 \\
.1 \mathrm{q} 26.2(161,540,639-168,592,236) \mathrm{hmz} \\
\operatorname{arr}[\mathrm{hg} 19] 7 \mathrm{p} 22 \\
.3 \mathrm{p} 21.2(2,707,569-13,857,235) \mathrm{hmz} \\
\operatorname{arr}[\mathrm{hg} 19] 8 \mathrm{q} 23 \\
.3 \mathrm{q} 24.12(114,788,423-119,897,611) \mathrm{hmz} \\
\operatorname{arr}[\mathrm{hg} 19] 9 \mathrm{p} 22 \\
.1 \mathrm{p} 13.3(19,696,747-36,125,149) \mathrm{hmz} \\
\operatorname{arr}[\mathrm{hg} 19] 11 \mathrm{q} 12 \\
.2 \mathrm{q} 12.3(60,193,879-63,210,491) \mathrm{hmz} \\
\operatorname{arr}[\mathrm{hg} 19] 11 \mathrm{p} 11 \\
.2 \mathrm{p} 11.21(45,781,075-51,550,787) \mathrm{hmz} \\
\operatorname{arr}[\mathrm{hg} 19] 17 \mathrm{q} 21 . \\
31 \mathrm{q} 21.32(41,647,165-44,927,874) \mathrm{hmz} \\
\operatorname{arr}[\mathrm{hg} 19] 17 \mathrm{q} 25 \\
.1 \mathrm{q} 25.3(71,965,953-75,785,426) \mathrm{hmz} \\
\operatorname{arr}[\mathrm{hg} 19] 20 \mathrm{p} 11 . \\
23 p 11.21(20,268,153-23,275,237) \mathrm{hmz}\end{array}$ & VUS & 77.5 & - & - \\
\hline
\end{tabular}


Table 4 (continued)

\begin{tabular}{|c|c|c|c|c|c|c|}
\hline Case & Indication & SNP array & Interpretation & Size $M b$ & Outcome & Inheritance \\
\hline 66 & $\begin{array}{l}\text { Thickened nuchal translucency, echogenic } \\
\text { intracardiac focus, mild tricuspid regurgi- } \\
\text { tation, hyperechogenic bowel }\end{array}$ & $\begin{array}{l}\operatorname{arr}[\mathrm{hg} 19] 18 \mathrm{q} 21 . \\
\quad 33 \mathrm{q} 22.1(60,147,532-65,974,912) \times 1 \mathrm{hmz}\end{array}$ & $\mathrm{P}$ & 5.8 & TP & - \\
\hline 67 & $\begin{array}{l}\text { Echogenic intracardiac focus, mild tricus- } \\
\text { pid regurgitation, pyelectasis }\end{array}$ & $\begin{array}{l}\operatorname{arr}[\mathrm{hg} 19] 6 \mathrm{6q21}(106,385,070- \\
\quad 109,072,236) \times 3\end{array}$ & VUS & 2.6 & TD & Denovo \\
\hline 68 & $\begin{array}{l}\text { Ventriculomegaly, hyperechogenic bowel, } \\
\text { echogenic intracardiac focus }\end{array}$ & $\begin{array}{l}\operatorname{arr}[\mathrm{hg} 19] \\
\quad 16 \mathrm{p} 11.2(28,810,324-29,032,280) \times 1\end{array}$ & $P$ & 0.2 & TP & - \\
\hline 69 & $\begin{array}{l}\text { Echogenic intracardiac focus, mild tricus- } \\
\text { pid regurgitation, short femur }\end{array}$ & $\begin{array}{l}\operatorname{arr}[\mathrm{hg} 19] 22 \mathrm{q} 11.21(21,059,669- \\
21,800,471) \times 1\end{array}$ & $P$ & 0.4 & TP & Denovo \\
\hline
\end{tabular}

$C N V$ s abnormal copy number variations, $P$ pathogenic, $T D$ term delivery, $T P$ termination of pregnancy, VUS variation of uncertain clinical significance

Therefore, SNP array is also recommended to examine fetuses with ultrasonic soft markers.

The rates of chromosomal abnormalities and abnormal CNVs in fetuses with short femur, thickened nuchal translucency, choroid plexus cyst, absent nasal bone, and ventriculomegaly are higher than those with other ultrasonic soft markers. Prenatal diagnosis is necessary for these fetuses, as reported in previous studies [12-14]. Chromosomal abnormalities were not found in fetuses with mild tricuspid regurgitation, hyperechogenic bowel, and echogenic intracardiac focus, but those of fetuses with abnormal CNVs were $12.5 \%, 4.6 \%$, and $4.5 \%$, respectively. According to reports $[15,16]$, mild tricuspid regurgitation occurred in $7 \%$ of normal fetuses. In this study, only 16 fetuses had mild tricuspid regurgitation, but two fetuses with mild tricuspid regurgitation had abnormal CNVs. Shuster and Keunen [17] considered abnormal CNVs in fetuses with hyperechogenic bowel. In this study, 65 fetuses with hyperechogenic bowel had normal karyotyping analysis results, but three fetuses with hyperechogenic bowel had abnormal CNVs. At present, many studies reported that fetuses with echogenic intracardiac focus did not have an increased risk for chromosomal abnormalities [18-20], but one pathogenic CNV was detected in 22 fetuses with echogenic intracardiac focus in this study. Therefore, SNP array should be considered when karyotyping analysis is carried out for fetuses with mild tricuspid regurgitation, hyperechogenic bowel, and echogenic intracardiac focus. In addition, no chromosomal abnormalities and abnormal CNVs were found in fetuses with right subclavian vagus, pyelectasis, single umbilical artery, and alteration of wave A in the ductus venosus. The sample size of fetuses with right subclavian vagus, pyelectasis, single umbilical artery, and alteration of wave A in the ductus venosus is relatively small; thus, more cases should be accumulated in the future.

Of the 69 abnormal CNVs, 37 were pathogenic CNVs. Although the clinical manifestations of patients with pathogenic CNVs are relatively mild compared with those with chromosomal aneuploidy, clinical manifestations of pathogenic CNVs cannot be ignored, as they can lead to various other clinical manifestations such as developmental delay, low intelligence, and autism [21]. 22q11.2 microdeletion syndrome, 22q11.2 microduplication syndrome, 3q29 microdeletion syndrome, and Williams-Beuren syndrome can lead to serious conditions such as stunting, mental retardation, and epilepsy, bringing heavy economic and mental burden to families. The application of SNP in prenatal diagnosis is particularly important.

Genetic abnormalities affect pregnancy outcomes. When the genomes of fetuses with ultrasonic soft markers are abnormal, their parents are more likely to terminate the pregnancy. In our study, pregnancies of 46 fetuses with chromosomal abnormalities, 37 with pathogenic CNVs, and three with VUS CNVs were terminated. SNP array can provide more objective theoretical basis for correctly evaluating the prognosis of fetus, and prenatal clinical counseling may help pregnant women decide on whether to continue the pregnancy.

SNP array improves the detection rate of chromosomal abnormalities, but SNP array can detect more VUS CNVs, which is a great challenge to genetic counseling. In this study, the detection rate of VUS CNVs was $2.8 \%$. In many cases, even after comparison of SNP array results of the parents, it is still impossible to accurately interpret the clinical characteristics of VUS. This may cause anxiety to pregnant women and even unnecessary termination of pregnancy [22-24]. Thus, a good working algorithm is necessary to try to minimize the uncertainty that the diagnosis of VUS.

\section{Conclusions}

In conclusion, we found an association between ultrasonic soft markers and genetic abnormalities in fetuses. Presence of ultrasonic soft markers, especially short femur, thickened nuchal translucency, choroid plexus cyst, absent nasal bone, ventriculomegaly, prenatal 
diagnosis and SNP array are recommended. The results of this study indicate that the genetic etiology of fetuses with ultrasonic soft markers is not only related to chromosomal abnormalities, but also related to abnormal CNVs. SNP array can complement for the deficiency of conventional karyotyping and improve the rate of chromosomal abnormalities. SNP array should be used for fetuses with ultrasonic soft markers and normal conventional karyotyping.

\section{Abbreviations \\ CNVs: Copy number variations; SNP: Single nucleotide polymorphism; VUS: Uncertain clinical significance.}

\section{Acknowledgements}

Not applicable.

\section{Authors' contributions}

LPX designed the study. MYC wrote the manuscript. HLH and NL revised the article. XMC, MMF, and NG performed the statistical analyses and interpreted the data. All authors have read and approved the final manuscript.

\section{Funding}

The present study was supported by the Fujian Provincial Natural Science Foundation, 2017J01238. The funder Hailong Huang designed the study and revised the article. The funding body provides support in data analysis.

\section{Availability of data and materials}

The microarray data from this study were submitted to the Gene Expression Omnibus repository (https://www.ncbi.nlm.nih.gov/geo/query/acc. cgi?acc=GSE163799).

\section{Ethics approval and consent to participate}

The study protocol was approved by the Ethics Committee of the Fujian Provincial Maternity and Children's Hospital (Fuzhou, China; approval no. 2014-042). Written informed consent was obtained from all participants or their guardians.

\section{Consent for publication}

Not Applicable.

\section{Competing interests}

The authors declare that they have no competing interests.

Received: 24 August 2020 Accepted: 1 January 2021

Published online: 12 January 2021

\section{References}

1. Ahman A, Axelsson O, Maras G, Rubertsson C, Sarkadi A, Lindgren P. Ultrasonographic fetal soft markers in a low-risk population: prevalence, association with trisomies and invasive tests. Ultrasound Obstet Gynecol. 2014;93(S1):367-73

2. Sagi-Dain L, Cohen Vig L, Kahana S, Yacobson S, Maya I. Chromosomal microarray vs. NIPS: analysis of 5541 low-risk pregnancies. Genet Med. 2019;21(11):1-6.

3. Li SL, Zhu J. Prenatal ultrasound diagnosis of fetal malformation. Beijing: People's Military Medical Publishing House; 2015.

4. Hanemaaijer NM, Sikkema-Raddatz B, van der Vries G, Dijkhuizen T, Hordijk R, Essen A, Veenstra-Knol H, Kerstjens-Frederikse W, Herkert J, Gerkes E. Practical guidelines for interpreting copy number gains detected by high-resolution array in routine diagnostics. Eur J Hum Genet. 2012;20(2):161-5.

5. Sohl BD, Scioscia AL, Budorick NE, Moore TR. Utility of minor ultrasonographic markers in the prediction of abnormal fetal karyotype at a prenatal diagnostic center. Am J Obstet Gynecol. 1999;181(4):898-903.
6. Wang J, Chen L, Zhou C, Liu H. Identification of copy number variations among fetuses with ultrasound soft markers using next-generation sequencing. Sci Rep. 2018;8(1):8134.

7. Bardin R, Hadar E, Haizler-Cohen L, Maya IR, Gabbay-Benziv I, Meizner S, Kahana J, Yeshaya S, Yacobson L, Cohen-Vig I, et al. Cytogenetic analysis in fetuses with late onset abnormal sonographic findings. J Perinat Med. 2018:46(9):975-82.

8. de Wit MC, Srebniak ML, Govaerts LC, Van Opstal D, Galjaard RJ, Go AT. Additional value of prenatal genomic array testing in fetuses with isolated structural ultrasound abnormalities and a normal karyotype: a systematic review of the literature. Ultrasound Obstet Gynecol. 2014;43(2):139-46.

9. Vestergaard EM, Christensen R, Petersen OB, Vogel I. Prenatal diagnosis: array comparative genomic hybridization in fetuses with abnormal sonographic findings. Acta Obstet Gynecol Scand. 2013;92(7):762-8.

10. Singletary CN, Krstic NC, Czerwinski JL, Wagner C. Prenatal chromosomal microarray uptake with invasive prenatal diagnosis: how many patients take the leap? Prenat Diagn. 2018;38(10):748-54.

11. Wapner RJ, Martin CL, Levy B, Ballif BC, Eng CM, Zachary JM, Savage M, Platt LD, Saltzman D, Grobman WA, et al. Chromosomal microarray versus karyotyping for prenatal diagnosis. N Engl J Med. 2012;367(23):2175-84.

12. Chong HP, Hamilton S, Mone F, Kilby MD. Prenatal chromosomal microarray testing of fetuses with ultrasound structural anomalies: a prospective cohort study of over 1000 consecutive cases. Prenat Diagn. 2019;39(12):1064-9.

13. Shah N. Prenatal diagnosis of choroid plexus cyst: what next? J Obstet Gynecol India. 2018;68(5):366-8.

14. Benacerraf BR, Bromley BS, Jelin AC. Absent nasal bone. Am J Obstet Gynecol. 2019;221(5):B6-7.

15. Wiechec M, Nocun A, Wiercinska E, Beithon J, Knafel A. First trimester tricuspid regurgitation and fetal abnormalities. J Perinat Med. 2015;43(5):597-603

16. Zhou J, Zhang Y, Gui Y, Chu C, Zhang C, Zhou Q, Li X, Yan Y. Relationship between isolated mild tricuspid valve regurgitation in second-trimester fetuses and postnatal congenital cardiac disorders. J Ultrasound Med. 2014;33(9):1677-82.

17. Shuster S, Keunen J, Shannon P, Watkins N, Chong K, Chitayat D. Prenatal detection of isolated bilateral hyperechogenic kidneys: Etiologies and outcomes. Prenat Diagn. 2019;39(9):693-700.

18. He M, Zhang Z, Hu T, Liu S. Chromosomal microarray analysis for the detection of chromosome abnormalities in fetuses with echogenic intracardiac focus in women without high-risk factors. Medicine. 2020;99(5):e19014.

19. Murphy H, Phillippi JC. Isolated intracardiac echogenic focus on routine ultrasound: implications for practice. J Midwifery Womens Health. 2015;60(1):83-8.

20. Lorente AMR, Moreno-Cid M, Rodriguez MJ, Bueno G, Tenias JM, Roman C, Arias A, Pascual A. Meta-analysis of validity of echogenic intracardiac foci for calculating the risk of Down syndrome in the second trimester of pregnancy. Taiwan J Obstet Gynecol. 2017;56(1):16-22.

21. Sansovic I, Ivankov AM, Bobinec A, Kero M, Barisic I. Chromosomal microarray in clinical diagnosis: a study of 337 patients with congenital anomalies and developmental delays or intellectual disability. Croat Med J. 2017:58(3):231-8.

22. Wapner RJ, Martin CL. Chromosomal microarray versus karyotyping for prenatal diagnosis. Obstet Gynecol Surv. 2013;68(4):2175.

23. Ganapathi M, Nahum O, Levy B. Prenatal diagnosis using chromosomal SNP microarrays. Methods Mol Biol. 2019;1885:187-205.

24. Pasternak Y, Daykan Y, Tenne T, Reinstein E, Miller N, Shechter-Maor G, Maya I, Biron-Shental T, Halevy RS. The yield of chromosomal microarray analysis among pregnancies terminated due to fetal malformations. J Matern Fetal Neonatal Med. 2020;23:1-5.

\section{Publisher's Note}

Springer Nature remains neutral with regard to jurisdictional claims in published maps and institutional affiliations. 